Article

\title{
Characterization of Lactate Sensors Based on Lactate Oxidase and Palladium Benzoporphyrin Immobilized in Hydrogels
}

\author{
Liam P. Andrus ${ }^{1}$, Rachel Unruh ${ }^{1}$, Natalie A. Wisniewski ${ }^{2}$ and Michael J. McShane ${ }^{1,3, *}$ \\ 1 Department of Biomedical Engineering, 5045 Emerging Technologies Building, 3120 TAMU, \\ Texas A\&M University, College Station, TX 77843, USA; E-Mails: lpa6@tamu.edu (L.P.A.); \\ rachel_unruh@tamu.edu (R.U.) \\ 2 PROFUSA, Inc., 345 Allerton Avenue, South San Francisco, CA 94080, USA; \\ E-Mail: natalie.wisniewski@profusa.com \\ 3 Department of Materials Science and Engineering, 3003 TAMU, Texas A\&M University, \\ College Station, TX 77843, USA \\ * Author to whom correspondence should be addressed; E-Mail: mcshane@tamu.edu; \\ Tel.: +1-979-845-7941; Fax: +1-979-845-4450.
}

Academic Editor: A. Sheila Holmes-Smith

Received: 30 April 2015 / Accepted: 24 June 2015 / Published: 7 July 2015

\begin{abstract}
An optical biosensor for lactate detection is described. By encapsulating enzyme-phosphor sensing molecules within permeable hydrogel materials, lactate-sensitive emission lifetimes were achieved. The relative amount of monomer was varied to compare three homo- and co-polymer materials: poly(2-hydroxyethyl methacrylate) (pHEMA) and two copolymers of pHEMA and poly(acrylamide) (pAam). Diffusion analysis demonstrated the ability to control lactate transport by varying the hydrogel composition, while having a minimal effect on oxygen diffusion. Sensors displayed the desired dose-variable response to lactate challenges, highlighting the tunable, diffusion-controlled nature of the sensing platform. Short-term repeated exposure tests revealed enhanced stability for sensors comprising hydrogels with acrylamide additives; after an initial "break-in" period, signal retention was $100 \%$ for 15 repeated cycles. Finally, because this study describes the modification of a previously developed glucose sensor for lactate analysis, it demonstrates the potential for mix-and-match enzyme-phosphor-hydrogel sensing for use in future multi-analyte sensors.
\end{abstract}


Keywords: luminescence; lactate; enzyme; biosensor; hydrogel

\section{Introduction}

Sensors capable of rapid and accurate analyte detection are essential to the future of personalized medicine [1,2]. Further, the ability to track and record in vivo substrate concentrations in real time will enable more timely, precise diagnosis and improved management of chronic conditions [3,4]. Currently, a blood sample is collected and analyzed ex vivo using an electrochemical enzyme assay. Even with the advent of portable, handheld, and point-of-care devices, patients endure painful, repeated fingersticks and the limitations of discrete time-point analysis do not provide caregivers or patients the ability to monitor systemic fluctuations over time. A prime example of this is diabetes, where knowledge of blood glucose dynamics helps inform decisions about treatment and lifestyle; yet, there are other situations where tracking biochemistry may also lead to improved outcomes.

For example, L-Lactate is an analyte of great interest due to its role in sports medicine [5], clinical chemistry [6], the food processing industry [7], and overall normal metabolic function. While lactate is a normal byproduct of cellular metabolism, intracellular concentrations of lactate increase more dramatically during anaerobic respiration; likewise, interstitial lactate levels increase as it is excreted by cells, and it can accumulate in muscles and other tissues to cause soreness, pain, and impaired function [8,9]. Therefore, lactate can be used to assess a variety of acute deoxygenation events including hypovolemia (shock) [10], heart disease [11], and renal failure [12]. High lactate levels are also commonplace in traumatic injury, where a patient has undergone significant blood loss. In fact, monitoring of blood lactate levels is shown to improve identification of patients requiring resuscitative care (93\%) when compared to standard blood pressure monitoring (67\%) [13]. Thus, continuous monitoring of lactate is attractive for dynamic health assessment of active-duty military and other high-risk personnel. Additionally, lactate levels can be used to approximate "oxygen debt" in endurance athletes [14], allowing for optimal training in their chosen endeavor. These facts highlight the use of continuous lactate monitoring for systemic health analysis in military personnel and other critical care situations $[13,15]$.

Although there have been recent advances in continuous analyte monitoring systems, there still remains a dearth of long term, minimally invasive options. To date, there have been a number of attempts to develop miniaturized platforms for continuous monitoring of various biochemical metabolites [16-20]. Many of these devices rely on an electrochemical-sensing assay for metabolite detection. For example, a successful system uses an enzyme to produce hydrogen peroxide $\left(\mathrm{H}_{2} \mathrm{O}_{2}\right)$ at the surface of an implanted electrode and the resulting change in electric potential can be correlated to interstitial lactate concentrations [21].

While electrochemical biosensors represent a major advancement in continuous interstitial analyte monitoring, they still experience a multitude of technical challenges to effective long-term use [22-25]. The entry wound caused by implantation elicits an inflammatory response from surrounding tissue. Macrophage recruitment eventually gives way to fibrotic encapsulation, restricting substrate diffusion at the tissue/electrode surface. These changes in sensitivity result in a reduction in the apparent 
sensitivity, requiring multiple recalibrations and eventually leads to shortened sensor lifetime, even though the sensors themselves retain function. In addition, the implantation site provides a potential bacterial pathway, leading to an increased risk of infection.

Optical sensing techniques display many advantages over electrochemical-based methods. Optical systems rely on photon transfer and detection, eliminating need of a transcutaneous connection. Previous work has shown the feasibility of using dual wavelength polarimetry [26], optical coherence tomography [27], and Raman spectroscopy [28] as techniques for metabolite detection and protein-protein interactions. Those studies, and many failed commercialization efforts, have also revealed the weakness of purely noninvasive approaches; they are not sensitive or selective enough to function effectively in real world situations.

As with optical glucose sensors, reports on optical lactate detection primarily use fluorescent indicator molecules [29,30] or spectroscopic [31] techniques. Molecular recognition agents provide a degree of selectivity for the target analyte, and luminescence detection dramatically improves sensitivity; the combination of these two properties results in "transducers" to convert chemical concentration into a fluorescent or phosphorescent signal [32]. The McShane group developed enzymatic glucose sensors based on microparticles and microcapsules with specialized diffusion-limiting coatings; Kazakova et al. developed lactate sensitive micro-capsules with a similar approach, using a $\mathrm{pH}$-sensitive fluorophore for detection [29]. In a similar approach, $\mathrm{Hu}$ et al. developed highly fluorescent cupric oxide nano-particles functionalized with terephthalic acid, a substrate in peroxidase catalysis. This allows for enzyme mimetic characteristics, albeit with more stable activity than seen in natural peroxidase. By coupling this $\mathrm{H}_{2} \mathrm{O}_{2}$ sensing system with a lactate consuming enzyme, a highly fluorescent lactate nano-sensor was created [30].

The aforementioned optical sensing modalities rely on changes in photon intensity and are thus susceptible to tissue scattering effects as well as variations in temperature, $\mathrm{pH}$ and other physiological variables. Both lactate sensors suffered from low effective ranges and their long-term stability was not evaluated, and reversibility is not possible in the peroxide-coupled system. Further, neither nano-particles nor micro-capsules demonstrated potential for use as an implantable device for continuous monitoring in vivo.

Recently, highly sensitive phosphors have been developed and applied for biomolecular assay applications [22]. Some of these molecules are sensitive to oxygen quenching. In ambient conditions, emission intensities remain low due to de-excitation via energy transfer to $\mathrm{O}_{2}$ by collisional quenching. As $\mathrm{O}_{2}$ is reduced, phosphorescent intensities and emission lifetimes increase significantly. This quenching behavior is described by the Stern-Volmer relationship.

$$
\frac{\tau_{0}}{\tau}=\frac{I_{0}}{I}=1+K_{s v}\left[\mathrm{O}_{2}\right]
$$

where $\tau_{0}$ and $I_{0}$ are phosphorescent lifetime and intensity of phosphor in absence of $\mathrm{O}_{2}, \tau$ and $I$ are lifetime and intensity, $K_{s v}$ is the phosphor specific Stern-Volmer constant, and $\left[\mathrm{O}_{2}\right]$ is oxygen concentration. Benzoporphyrin-based oxygen indicators absorb and emit at wavelengths within the commonly termed "optical window", allowing for relatively efficient photon transmission through skin [33-36]. Additionally, phosphors are characterized by relatively long emission lifetimes (single to hundreds of microseconds), compared to tissue fluorophores (few nanoseconds); this property can be 
utilized to effectively discriminate sensor emission from background and natural fluorescence using straightforward signal processing techniques. Thus, emission lifetime can be used in lieu of intensity as a means to sensitively interrogate implants with minimal interference.

Oxygen-sensitive phosphors can be exploited for alternative substrate analysis by co-localization with an oxidoreductase enzyme. One example, lactate oxidase (LOx), uses both $\mathrm{O}_{2}$ and lactate as its co-substrates. Kinetics of LOx are described in the following reaction equations.

$$
\begin{gathered}
E_{\text {ox }}+L \stackrel{k_{1}}{\Rightarrow} E_{\text {red }} X_{1} \stackrel{k_{2}}{\Rightarrow} E_{\text {red }}+P \\
E_{\text {red }}+\mathrm{O}_{2} \stackrel{k_{3}}{\Rightarrow} E_{\text {ox }}^{*} X_{2} \stackrel{k_{4}}{\Rightarrow} E_{o x}+\mathrm{H}_{2} \mathrm{O}_{2}
\end{gathered}
$$

where $E_{o x}$ and $E_{\text {red }}$ are the oxidized and reduced form of LOx, $L$ is lactate, $P$ is pyruvate, $E_{r e d} X_{1}$ is reduced enzyme-substrate complex, $E^{*}{ }_{o x} X_{2}$ is oxidized enzyme-substrate complex, and $k_{1}, k_{2}, k_{3}, k_{4}$ are reaction rate constants (only forward constants are shown here) [37]. Incoming lactate increases catalysis and consequently oxygen consumption. Less collisional quenching by molecular $\mathrm{O}_{2}$ leads to longer emission lifetimes of the co-localized phosphor. Thus, approximation of local lactate levels is possible by monitoring phosphor quenching kinetics in an enzyme-controlled $\mathrm{O}_{2}$ micro-environment.

One key to using this concept to create implantable sensor devices is the encapsulation of the enzyme and phosphor sensing components into an appropriate biomaterial matrix, such as a hydrogel [38]. Poly(2-hydroxyethyl methacrylate) (pHEMA) is a synthetic polymer used in numerous biomedical devices, most notably soft contact lenses [39], drug delivery systems [40,41], and tissue engineering constructs [42]. pHEMA is attractive for use in implantable devices due to its low cytotoxicity and biofouling properties [43]. pHEMA's ability to swell in water without losing mechanical integrity makes it an ideal material for in vivo applications [44-47]. Similarly, poly(acrylamide) (pAam) has many uses, initially purposed as a separation medium for gel electrophoresis applications $[48,49]$. Like pHEMA, pAam's hydrophilic nature and low biofouling properties make it suitable for in vivo applications [49]. Additionally, both pHEMA and pAam have been investigated for immobilization of enzymes and other indicator molecules in biosensing applications [50-52]. Cross-linking enzymes within hydrogels has shown to enhance catalytic stability [53-55]. Thus, homogenous distribution of sensing chemistry within a biocompatible gel is attractive for enzyme based sensing applications.

This paper reports on in vitro characterization of a hydrogel based phosphorescent biosensor for lactate determination, where the system is designed to meet the needs described above. The studies herein focus on determining the effects of co-localizing LOx and an oxygen-sensitive phosphor within a hydrogel matrix, particularly on the oxygen and lactate diffusion, dose-response of the entire system to lactate (oxygen depletion), and finally the stability of the sensors to repeated cycling. This builds upon our previous efforts with glucose sensing, illustrating the general applicability of the enzyme-benzoporphyrin-hydrogel platform and how it may be used to take us toward the ultimate goal of multianalyte detection. 


\section{Experimental Section}

\subsection{Materials}

Catalase, ethylene glycol, 2,2-dimethoxy-2-phenyl-acetophenone (DMAP), 1-Ethyl-3-[3dimethylaminopropyl]carbodiimide hydrochloride (EDC), and sodium lactate were purchased from Sigma-Aldrich $^{\circledR}$ (St. Louis, MO, USA). Dimethyl sulfoxide (DMSO) was purchased from VWR ${ }^{\circledR}$ (Radnor, PA, USA). Sodium chloride, potassium phosphate (dibasic), and potassium chloride were purchased from VWR (Radnor, PA, USA). Sodium phosphate (monobasic) was purchased from

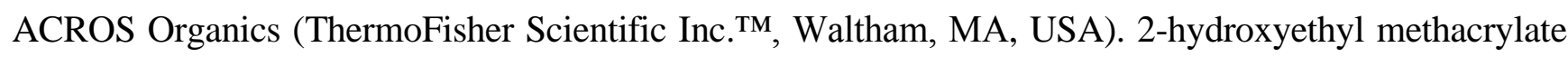
(HEMA) and tetra(ethylene glycol) methacrylate (TEGDMA) were purchased from Polysciences Inc. (Warrington, PA, USA). Lactate oxidase from Aerococcus viridians (LOx), acrylamide (Aam), and sulfo- $N$-hydroxysuccinimide (sulfo-NHS) were purchased from A.G. Scientific ${ }^{\mathrm{TM}}$ (San Diego, CA, USA), AMRESCO $^{\circledR}$ (Solon, OH, USA), and G Biosciences ${ }^{\mathrm{TM}}$ (St. Louis, MO, USA) respectively. Palladium (II) tetramethacrylated benzoporphyrin (BMAP) was donated by PROFUSA Inc., (San Francisco, CA, USA). All chemicals are reagent grade and used without further purification.

\subsection{Sensor Preparation}

To synthesize gels, $2.5 \mathrm{mg}$ DMAP was weighed into a micro centrifuge tube. Aam was dissolved in DMSO in a $67.2 v / v \%$ concentration. Two hundred and fifty microliters of monomer precursor (containing the proper $v / \nu \%$ ratio of HEMA to Aam solution plus $5 \mu \mathrm{L}$ TEGDMA) was added to DMAP and vortexed. Next, $90 \mu \mathrm{L}$ ethylene glycol was added as a co-solvent and vortexed again. Fifty microliters of $10 \mathrm{mM}$ BMAP solution in DMSO was added along with $125 \mu \mathrm{L}$ LOx/Catalase solution $(\mathrm{pH}=7.4)$ in a 10:1 molar ratio. Both dye and enzyme were repeatedly pipetted to ensure proper mixing. Sensors were made with three different co-polymer materials: 75:25 pHEMA:pAam, 90:10 pHEMA:pAam and pure pHEMA. Resulting solutions were pipetted into a premade mold (consisting of a $0.03^{\prime \prime}$ spacer sandwiched between two clean microscope slides) and exposed to UV light to induce polymerization. Gels were removed from molds and placed into PBS solution containing $15 \mathrm{mg}$ sulfo-NHS and $6.6 \mathrm{mg}$ EDC. To ensure maximum enzyme cross-linking within gels, sensors were allowed to react overnight. Afterwards, gels were rinsed with DI water, placed in fresh PBS, and stored at $4{ }^{\circ} \mathrm{C}$ in foil to prevent photobleaching. Sensors were cut into $5 \mathrm{~mm}$ strips rinsed prior to testing.

\subsection{Benchtop Testing System}

To validate in vitro sensor response, a custom flow-through system was used. Two positive displacement VICI ${ }^{\circledR}$ M6 liquid pumps (Valco Instruments Co., Inc., Houston, TX, USA) are connected to reservoirs containing either a highly concentrated (20 mM lactate) solution or a PBS solution ( $0 \mathrm{mM}$ lactate). A LabVIEW ${ }^{\mathrm{TM}}$ program controls flow rates from both pumps independently. Flow from each reservoir is mixed prior to reaching a specially designed cell in which four sensors are immobilized. In a typical experiment, three lactate sensors and a pHEMA-BMAP oxygen sensor were placed in the flow cell for simultaneous monitoring. Lactate concentration was modulated throughout each experiment 
and resulting lifetime data was collected and stored for later analysis. It was deemed impractical to test sensors in blood or serum at this time, due to contamination of pumps in addition to the volume of solutions needed. While the conditions used for testing do not completely represent the in vivo expected situation, they should be considered adequate for the initial characterization of this new type of sensor system. A schematic of the flow through system is shown in Figure 1.

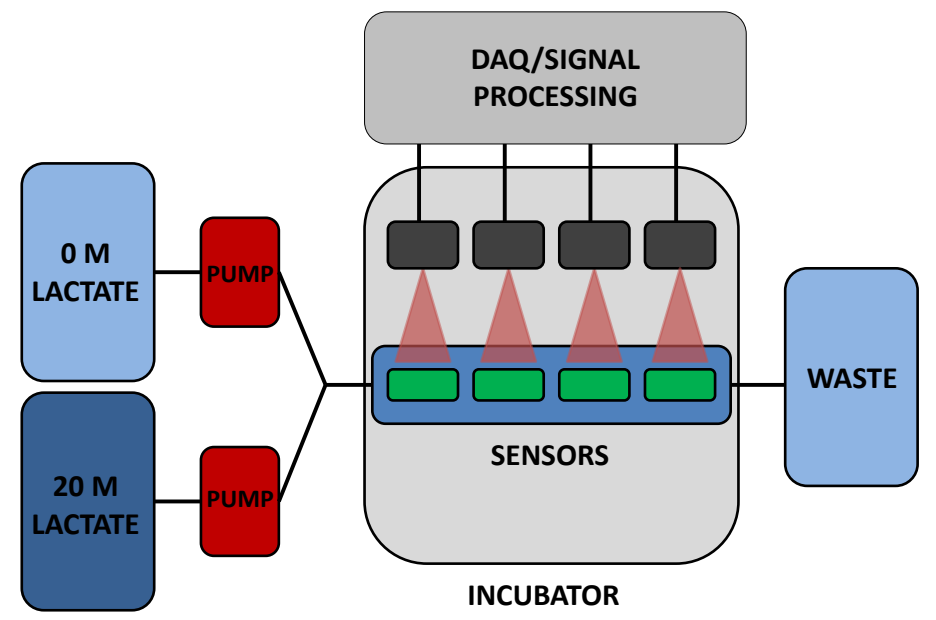

Figure 1. Flow through system schematic.

To interrogate immobilized BMAP, a custom optical instrument was used. The excitation component of the setup is a Phillips ${ }^{\circledR}$ Lumileds Luxeon Rebel red excitation source with a peak at $\lambda_{\text {typ }}=627 \mathrm{~nm}$ and a bandwidth of $\approx 20 \mathrm{~nm}$. The diode has a $125^{\circ}$ viewing angle, common among most LEDs, and is operated at $200 \mathrm{~mA}$. Upon phosphor excitation, emitted photons are collected with a ball lens situated at $8 \mathrm{~mm}$ distance from the LED port; this light is collimated and passed through a Semrock ${ }^{\mathrm{TM}}$ FF02-809/81 filter prior to detection with a Si PMT (SensL). The entire system is contained within a miniaturized 1.5" diameter plastic casing; each such "reader head" interrogates a single sensor of interest. A LabVIEW ${ }^{\mathrm{TM}}$ program controls LED intensity, pulse on/off parameters, data acquisition, and emission lifetime calculation from the measured decays as described below.

\subsection{Data Analysis and Sensor Response}

Emission lifetime of immobilized phosphor was measured as decay in voltage signal across a PMT detector. Immediately following a $500 \mu$ s LED pulse, peak intensity was measured. Sampling of voltage was delayed for $7 \mu$ s after pulse to reduce interference from the LED excitation pulse. Background voltage was calculated as the average intensity of the last $20 \%$ of the decay duration $(2500 \mu \mathrm{s})$. Acquisitions were repeated quickly, generally taking 1.3 seconds per cycle. Emission lifetime decay curves were found by averaging 128-256 consecutive sets of intensity decays. Decay signal is defined from $t=507 \mu$ s to $t=2500 \mu \mathrm{s}$. Data were fit to an exponential defined in Equation (3). From this a value for phosphorescent lifetime $(\tau)$ may be obtained.

$$
[Q]_{t}=[Q]_{0} \mathrm{e}^{-t / \tau}
$$


where $[Q]_{t}$ and $[Q]_{0}$ are concentrations of excited state molecules at time $t$ and time 0 , respectively, and $\tau$ is the phosphorescent lifetime. Excited photons are lost due to various radiative and non-radiative processes. This makes the total phosphorescent lifetime a sum of each individual contribution.

A number of commonly used performance metrics were used to assess sensor response. After exposing sensors to an initial lactate challenge, adjustments were made to modulate sensors within a more appropriate range. Specifically, the lactate concentration steps used in the challenges were adjusted to provide for calibration curves with many points where the responses were most sensitive. Analytical range, sensitivity, and response times were calculated from calibration curves of $n=3$ sensors. Analytical range is the span over which sensors accurately detect changes in lactate concentration, defined as the interval between upper and lower limits of detection ( $\left.\mathrm{LOD}_{\text {high }} / \mathrm{LOD}_{\text {low }}\right)$. $\mathrm{LOD}_{\text {low }}$ is defined as emission lifetime seen at $0 \mathrm{mg} / \mathrm{dL}$ lactate plus (+) 3 standard deviations. Similarly, $\mathrm{LOD}_{\text {high }}$ is defined as emission lifetime at enzyme saturation minus (-) 3 standard deviations. Sensitivity is defined as the linear slope of each calibration curve, while response time refers to lag between lactate input and steady sensor response. Each point on a calibration curve represents a steady lifetime response over $30 \mathrm{~min}$ for a given lactate concentration. While steady response was observed within the first twenty minutes of a step increase in lactate, we allowed sensors to be exposed to each concentration for $1 \mathrm{~h}$ in order to collect more data points, thus providing for more robust data analysis. Sensors were subjected to a flow rate of $4 \mathrm{~mL} / \mathrm{min}$. "Flight plans" are progressively higher lactate challenges run consecutively on sensors before a return to baseline. Unless otherwise noted, all lactate challenges were performed at $37^{\circ} \mathrm{C}$.

\subsection{Diffusion Analysis}

To assess lactate transport, a horizontal diffusion cell system was used, following procedures to those detailed in previous work [52]. Briefly, hydrogels were prepared as described in Section 2.2, except that polymerization was performed using a $0.01^{\prime \prime}$ glass mold; the thinner samples allowed for more rapid experimentation. Sensors were preconditioned in a $60{ }^{\circ} \mathrm{C}$ water bath for 10 days prior to diffusion study in order to completely deactivate immobilized LOx. LOx deactivation was confirmed by monitoring phosphorescent signal during a single lactate challenge. Deactivation of LOx mitigates lactate consumption concerns and allowed for a more reliable analysis of diffusion kinetics.

Each 7-mm diameter gel cutouts was sandwiched between two $7 \mathrm{~mL}$ reservoirs. Feeder reservoirs contain $1 \mathrm{M}$ lactate solution, while permeate reservoirs contain pure PBS solution. To determine the rate of concentration change, $d c / d t$, permeate chambers were sampled for lactate over several hours using a YSI ${ }^{\mathrm{TM}} 2700$ Select Biochemistry Analyzer (YSI Inc ${ }^{\mathrm{TM}}$, Yellow Springs, OH, USA); $d c / d t$ was estimated as the slope of a linear fit to the concentration data. To calculate a relative diffusion coefficient, $D_{L}$, Fick's 2nd law of diffusion was used, with the assumption that $c_{l a c}(0)=$ at $t(0)$ in the permeate reservoir and homogenous mixing in each,

$$
D_{L}=\frac{d c}{d t}\left(\frac{b V}{\emptyset}\right)
$$

where $D_{L}$ is the diffusion coefficient of lactate in $\mathrm{cm}^{2} / \mathrm{s}, d c / d t$ is change in permeate reservoir concentration over time, $b$ is thickness of gel, $V$ is the ratio of volume of reservoir to area of gel 
exposed to solution, and $\phi$ is the partition coefficient (assumed to be 1). Every material type was tested in triplicate at $25^{\circ} \mathrm{C}$ and $D_{L}$ is reported as an average.

$\mathrm{O}_{2}$ transport was investigated by analyzing quenching kinetics of immobilized BMAP. Using Equation (1), material specific $K_{s v}$ values were calculated. Six hundred milliliters of PBS solution is connected to a flow cell containing sensors. Dissolved $\mathrm{O}_{2}$ concentration in PBS solution is controlled with a nitrogen bubbling system. A 1179A, MKS mass flow controller and a pressure gauge controller model PR 4000F, MKS, (MKS Instruments, Andover, MA, USA) are used to control flow of nitrogen gas and compressed air to the bubbler probe. $\mathrm{O}_{2}$ was decreased in a stepwise manner at concentrations of $21 \%, 10.5 \%, 5.25 \%$, and $2.1 \%$. Afterwards, $0 \% \mathrm{O}_{2}$ was achieved by loading flow cell with $4 \mathrm{M}$ glucose and $30 \mu \mathrm{M}$ GOx solutions. The flow cell was then sealed and the mixture was allowed to react overnight. The resulting reaction consumes all local $\mathrm{O}_{2}$, allowing for detection of $\tau_{0}$. Stern-Volmer constants, $K_{s v}$, were calculated from a linear fit to the calculated $\tau_{0} / \tau$. Each sensor type was tested in triplicate at $37{ }^{\circ} \mathrm{C}$.

\subsection{Acute Sensor Degradation}

To test for acute enzyme degradation, sensors were subjected to 20 consecutive lactate challenges. Flight plans were normalized in order to maintain a similar rate of enzymatic consumption within each material, meaning concentrations used were determined for each sensor type based on the upper limit of detection. Interrogations are step increases in lactate concentration before a return to baseline as seen in Section 2.4; however flight plans modulate between $0 \mathrm{mg} / \mathrm{dL}$ and the predetermined $\mathrm{LOD}_{\text {high }}$ of each sensor iteration. "Flight plans" were executed in series, with a return to $0 \mathrm{mg} / \mathrm{dL}$ lactate in between each. Afterwards, signal loss was calculated as percent change in emission lifetime from the initial (cycle 1) flight plan. Percent change was calculated at $\mathrm{LOD}_{\text {high }}$ as well as at intermediate concentrations.

\section{Results and Discussion}

\subsection{Diffusion Analysis}

Figure 2a contains the measured permeate chamber lactate concentration for the three sensor types. Qualitatively, the increase in lactate transport through gels containing acrylamide is obvious. The calculated relative $D_{L}$ values for 75:25 pHEMA:pAam, 90:10 pHEMA:pAam, and pure pHEMA are $5.68 \pm 0.323 \times 10^{-7}, 3.98 \pm 0.97 \times 10^{-7}$, and $3.13 \pm 1.62 \times 10^{-7} \mathrm{~cm}^{2} / \mathrm{s}$, respectively. 90:10 pHEMA:pAam sensors display a small increase in lactate transport when compared to pure pHEMA, suggesting more pAam is needed in order to substantially effect swelling properties. For 75:25 pHEMA:pAam sensors, $\mathrm{a} \approx 2$-fold increase in lactate diffusion is seen relative to pHEMA sensors.

Figure $2 \mathrm{~b}$ contains $\tau_{0} / \tau$ vs. $\left[\mathrm{O}_{2}\right]$ plots for each material tested. $K_{s v}$ values for 75:25 pHEMA:pAam, 90:10 pHEMA:pAam, and pure pHEMA are $0.29 \pm 0.003\left(\% \mathrm{O}_{2}\right)^{-1}, 0.28 \pm 0.01\left(\% \mathrm{O}_{2}\right)^{-1}$, and $0.28 \pm 0.002\left(\% \mathrm{O}_{2}\right)^{-1}$, respectively. These $K_{s v}$ values match well with previous studies on similar materials [35]. Only the pure pHEMA and 75:25 formulations were statistically different at the 95\% confidence level; this difference, while statistically significant, is only a matter of $3.6 \%$ increase in oxygen quenching. Thus, the effects on oxygen diffusion are minimal. This is not surprising, as $\mathrm{O}_{2}$ is a very small, hydrophobic molecule with low solubility in water. Thus, transport may depend less on 
material cross-link density and more on the $\mathrm{O}_{2}$ favorability of each polymer. It is most important to appreciate here that the changes in polymer formulation dramatically change lactate diffusion while minimally altering oxygen diffusion. Thus, this particular combination of polymers allows tuning of lactate diffusion almost completely independently from oxygen.

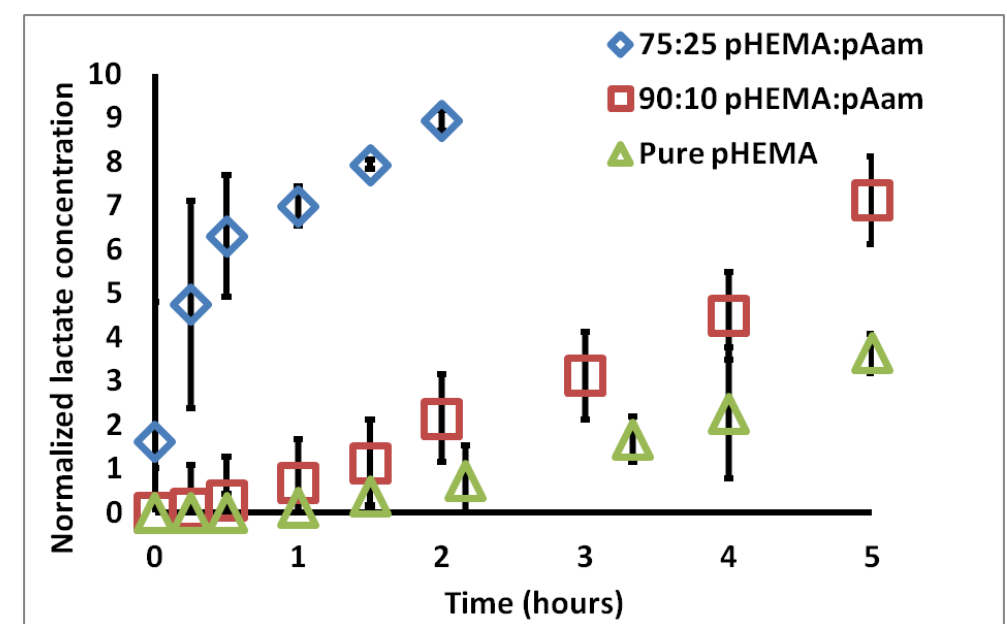

(a)

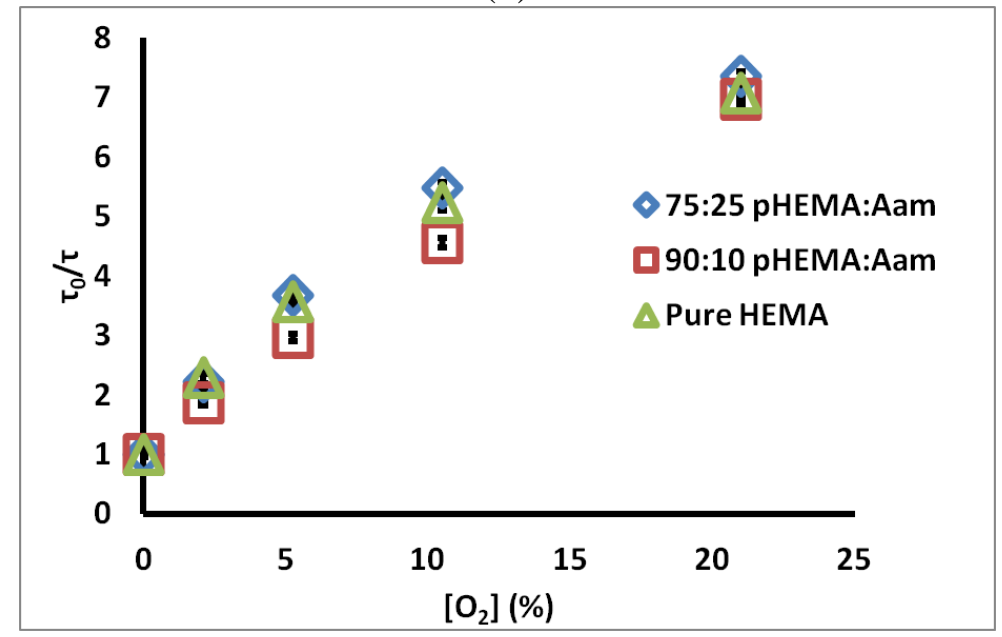

(b)

Figure 2. (a) Change of lactate concentration in permeate chamber over time for three sensor types; (b) Stern-Volmer plots for the same sensor types. Each set is an average of three compositionally identical sensors; errors bars denote $95 \%$ confidence intervals.

\subsection{Sensor Response}

To determine characteristic sensor response, lactate challenges were administered. Figure 3a contains a representative real-time "flight plan" plot of the change in phosphorescent lifetime for three 75:25 pHEMA:pAam sensors to progressively higher lactate concentrations. The observed stepwise response is common to all formulations, regardless of composition. Most sensors matched well with others from the same batch, while a few cases (such as Channel 2 in Figure 3a) were significantly different in their response to intermediate lactate concentrations. All lactate sensors plateaued at a maximum lifetime between 225 and $250 \mu \mathrm{sec}$. A fourth trace represents the response of the oxygen 
sensor placed in the same channel, indicating the stable oxygen level observed in the steady state even during lactate challenges.

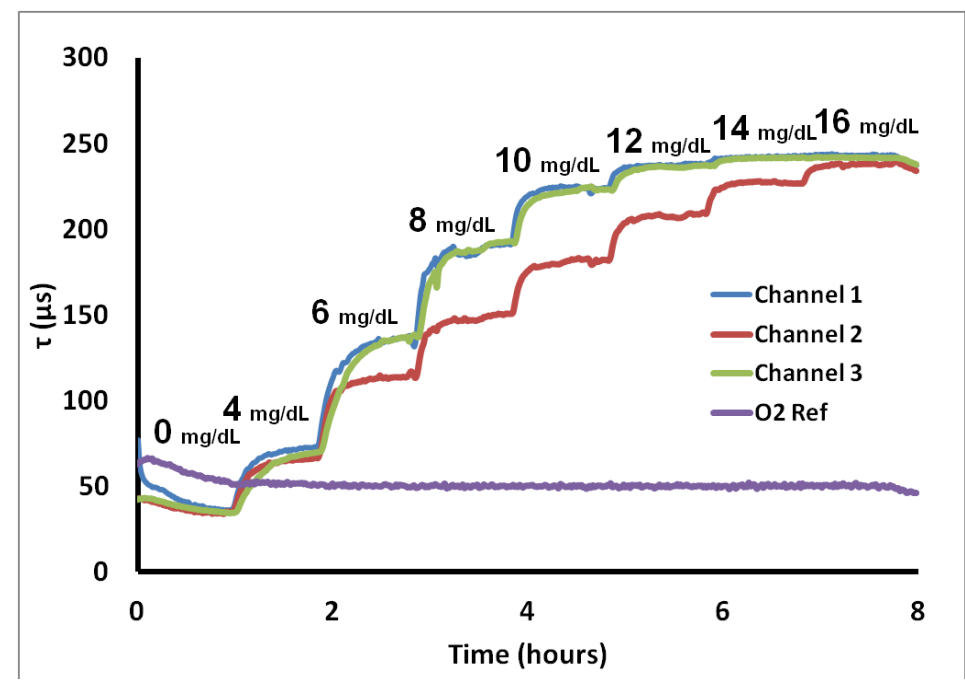

(a)

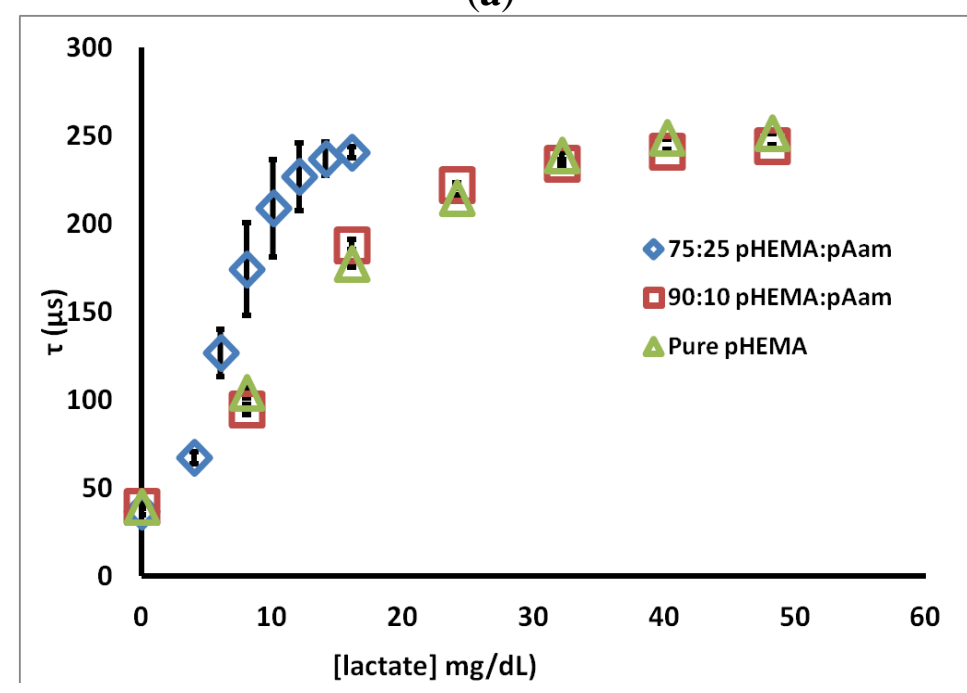

(b)

Figure 3. (a) 75:25 pHEMA:pAam lifetime response to lactate interrogation (b) calibration curves for three sensor types. Each calibration curve contains points representing the average phosphorescent lifetime; error bars denote the $95 \%$ confidence intervals for $n=3$ sensors.

Figure $3 \mathrm{~b}$ shows calibration curves representing the steady-state response to lactate for sensors based on the three different hydrogel types. Several points can be made from these data. First, all of the formulations yield the same lifetime at zero lactate, again reinforcing that the oxygen diffusion properties are essentially the same for each case. It is also immediately apparent that the incorporation of $25 \%$ acrylamide dramatically shifts the sensor behavior from the other two cases. First, the sensitivity to lower lactate levels was increased, while the lactate concentration at which lifetime nears the maximum ( $\mathrm{LOD}_{\text {high }}$ ) was cut in approximately half. Furthermore, the amount of variability between sensors increased significantly, as indicated by the larger confidence intervals. This increased variability is primarily a result of batch heterogeneity, which was observed visually in preparing the hydrogels. The increased acrylamide content resulted in an obvious increase in gel phase 
separation/heterogeneity. Thus, sensors cut from the same initial hydrogel slab are more inconsistent in appearance. This apparent difference likely results in the performance difference, which we attribute to variability in localized enzyme concentration and diffusion properties in the acrylamide-containing gels. This heterogeneity is less pronounced for the 90:10 copolymers. It should be noted that a number of options exist to reduce the batch variability seen herein. Use of solvent mixtures that improve miscibility of the complex precursor, thereby reducing aggregation/phase separation/precipitation of the sensing chemistry during the mixing and polymerization process, is one option. Additionally, enzyme modification techniques (i.e., PEGylation, modification of glycosylation sites with more hydrophilic or hydrophobic species) may be performed in order to allow for dissolution in alternative solvents without substantial activity loss. Lastly, precipitates may be filtered out prior to polymerization, if observed in the precursor mixtures. This latter option is an inefficient but viable solution.

Corresponding sensor figures of merit and diffusion metrics for the 75:25 pHEMA:pAam, 90:10 pHEMA:pAam, and pure pHEMA materials are reported in Table 1. These numbers quantitatively support the notion that increasing pAam precursor ratios are correlated to a decrease in range and a corresponding increase in sensitivity. This inverse relationship is explained by the properties of the two polymers. pAam is known to be significantly more hydrophilic than pHEMA due to polar amide groups present in pAam's primary structure. In fact, pAam is able to take on $\approx 80 \%$ its weight in aqueous solution (compared to $\approx 37 \%$ for pHEMA) [36]. Higher concentrations of pAam lead to a more loosely cross-linked matrix, and therefore a more rapid diffusion profile, as is clearly seen from the measured relative lactate diffusion values. In contrast, however, previous reports cited a much larger increase of diffusion when comparing pAam to pHEMA; diffusion of small molecules was been shown to be several orders of magnitude higher in pAam when compared to pure pHEMA [52]. Thus, the copolymer system retains a strong influence of the pHEMA even with $25 \%$ pAam.

Table 1. Compiled sensor metrics, values are average of three sensors $\pm 95 \%$ confidence intervals.

\begin{tabular}{cccc}
\hline Monomers & 75:25 pHEMA:pAam & 90:10 pHEMA:pAam & Pure pHEMA \\
\hline Sensitivity $(\mu \mathrm{s} \times \mathrm{dL} / \mathrm{mg})$ & $19.0 \pm 2.3$ & $9.2 \pm 1.5$ & $8.5 \pm 2.2$ \\
Range $(\mathrm{mg} / \mathrm{dL})$ & $1.1-12.7$ & $0.7-35.0$ & $0.4-38.2$ \\
Response time $(\mathrm{min})$ & $19.0 \pm 2.9$ & $16.4 \pm 1.7$ & $15.2 \pm 1.2$ \\
$D_{L}\left(\mathrm{~cm}^{2} / \mathrm{s}\right) \times 10^{-7}$ & $5.7 \pm 0.3$ & $4.0 \pm 0.9$ & $3.1 \pm 1.6$ \\
$\tau_{0}(\mu \mathrm{s})$ & $251.8 \pm 5.1$ & $259.9 \pm 8.9$ & $290.0 \pm 7.3$ \\
$K_{s v}\left(\%^{-1} \mathrm{O}_{2}\right) \times 10^{-2}$ & $29.4 \pm 0.3$ & $28.0 \pm 1.2$ & $27.7 \pm 0.2$ \\
Signal retention @ LOD & & & \\
For 0-20x cycles & & & \\
& & & \\
\end{tabular}

This change in diffusivity alters kinetics of immobilized LOx and the resulting oxygen consumption profiles. Increased swelling allows local lactate and $\mathrm{O}_{2}$ molecules to interact more readily with LOx active sites, encouraging more rapid enzyme saturation. As more $\mathrm{O}_{2}$ is consumed, BMAP is quenched less and therefore emits with a longer lifetime. Thus, the optical saturation is reached at lower bulk lactate concentrations. Higher pAam concentration increases enzyme-substrate contact, effectively lowering usable range of the device.

An interesting note is that $D_{L}$ values scale well with sensitivity metrics. Addition of only $10 \%$ pAam does little to increase lactate diffusion (and therefore sensitivity). pAam is much more hydrophilic 
than pHEMA, but is needed in higher ratios to significantly affect gel microstructure. Both sensitivity and $D_{L}$ metrics for the 75:25 pHEMA:pAam sensor are double what is seen in pure pHEMA gels, indicating promise for high measurement precision within a normal lactate range.

Response times calculated for sensor types were not statistically different between the materials. All materials were able to achieve a stable optical response within a 20 -min window after introducing the step change in lactate level. This is similar to response times for current electrochemical-based sensors [56,57] and is considered adequate to effectively monitor changes in systemic conditions.

The 90:10 pHEMA:pAam and pure pHEMA sensors cover the normal physiological lactate range $(0-30 \mathrm{mg} / \mathrm{dL})$. Comparing sensors with those found in the literature indicates a general improvement in performance, even for these "disconnected" devices that are noninvasively interrogated. Garjonyte et al. and Palmisano et al. developed LOx-based amperometric biosensors with linear responses up to 7.2 and $1.8 \mathrm{mg} / \mathrm{dL}$ lactate, respectively [58,59]. Ibupoto et al. developed an electrochemical sensor with a high $\mathrm{LOD}_{\text {high }}(90 \mathrm{mg} / \mathrm{dL})$ by immobilizing LOx on $\mathrm{ZnO}$ nanorods [60]. In regards to opticallybased lactate sensors, Marquette et al. developed a fluorescent lactate sensor by co-immobilizing LOx and luminol onto the end of an optical fiber, although the LOD $_{\text {high }}$ found was very low (30 pM) [61]. The system developed by $\mathrm{Hu}$ et al. had a calculated $\mathrm{LOD}_{\text {high }}$ of $45 \mathrm{nM}$ [30]. While this list is not exhaustive, it shows that our platform competes and in several cases outperforms other lactate sensing modalities. Importantly, the platform we have shown here displays significantly higher LODs when compared to other optically-based lactate sensors found in the literature. It is these that would be potentially competing with our approach for fully-implanted sensing materials, if they could be modified to function in such a manner. While a select number of electrochemical lactate sensors have higher upper LODs, they still require a physical connection to transmit a signal whereas our system does not.

\subsection{Acute Loss of Sensor Function}

To test for acute reduction in sensor response to lactate, sensors were exposed to 20 consecutive lactate challenges. Lifetime values at 5, 10, and 20 lactate challenges were compared against initial flight plan lifetimes for freshly prepared sensors. Signal retention was calculated as a metric of the change in response after repeated exposures by determining the difference between the initial response lifetimes (Cycle 1). This metric is described in Equation (5).

$$
\text { Signal retention }(\%)=\left[\frac{\left(\tau_{\text {cycle } x 20}-\tau_{\text {cycle } 01}\right)}{\tau_{\text {cycle } x 1}-\tau_{\text {cycle } 01}}\right] * 100
$$

where $\tau_{\text {cycle } x 20}$ is the emission lifetime at cycle $20, \tau_{\text {cycle } x 1}$ is lifetime for same concentration on the initial cycle, and $\tau_{\text {cycle } 01}$ is the baseline $\tau$ recorded at $0 \mathrm{mg} / \mathrm{dL}$ lactate for BMAP on the first cycle.

Figure 4a contains representative data from the 90:10 pHEMA:pAam materials, indicating how the measured lifetimes at each lactate concentration changed over 20 cycles. Figure $4 \mathrm{~b}$ is a summary of the signal retention over 20 cycles for all three sensor types. For 75:25 pHEMA:Aam sensors, signal retention of $78.1 \% \pm 9.0 \%, 76.1 \% \pm 18.4 \%, 73.1 \% \pm 14.9 \%$ is seen at $1 / 3 \mathrm{LOD}_{\text {high }}, 2 / 3 \mathrm{LOD}_{\text {high }}$, and $\mathrm{LOD}_{\text {high }}$, respectively. For 90:10 pHEMA:pAam sensors, signal retention of $85.5 \% \pm 20.8 \%$, $80.6 \% \pm 16.5 \%, 81.0 \% \pm 10.6 \%$ is seen at $1 / 3 \mathrm{LOD}_{\text {high }}, 2 / 3 \mathrm{LOD}_{\text {high }}$, and $\mathrm{LOD}_{\text {high }}$, respectively. Finally, 
pure pHEMA sensors signal retention of $34.9 \% \pm 11.8 \% .56 .1 \% \pm 8.0 \%, 69.9 \% \pm 4.9 \%$, is seen at 1/3 LOD $\mathrm{Ligh}_{\text {, }} 2 / 3 \mathrm{LOD}_{\text {high, }}$ and $\mathrm{LOD}_{\text {high }}$, respectively.

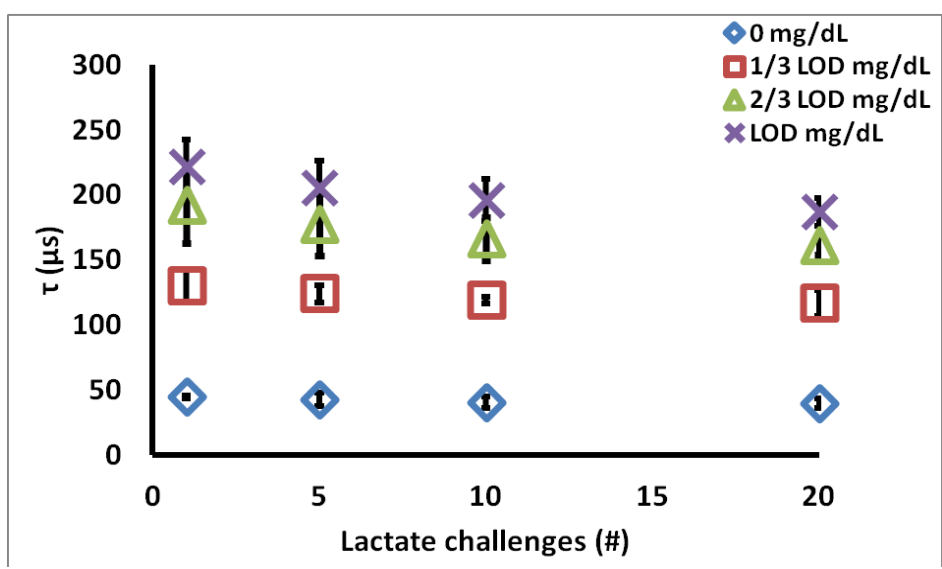

(a)

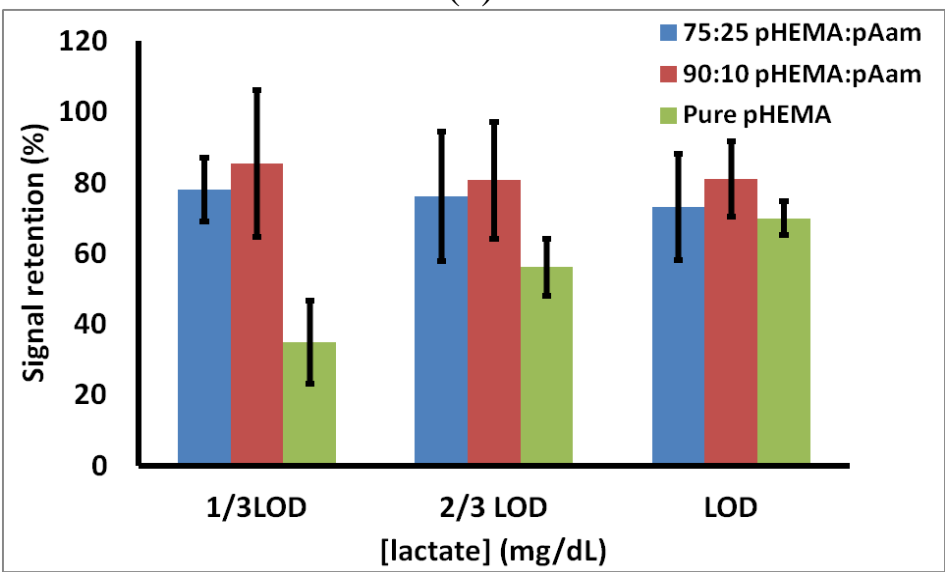

(b)

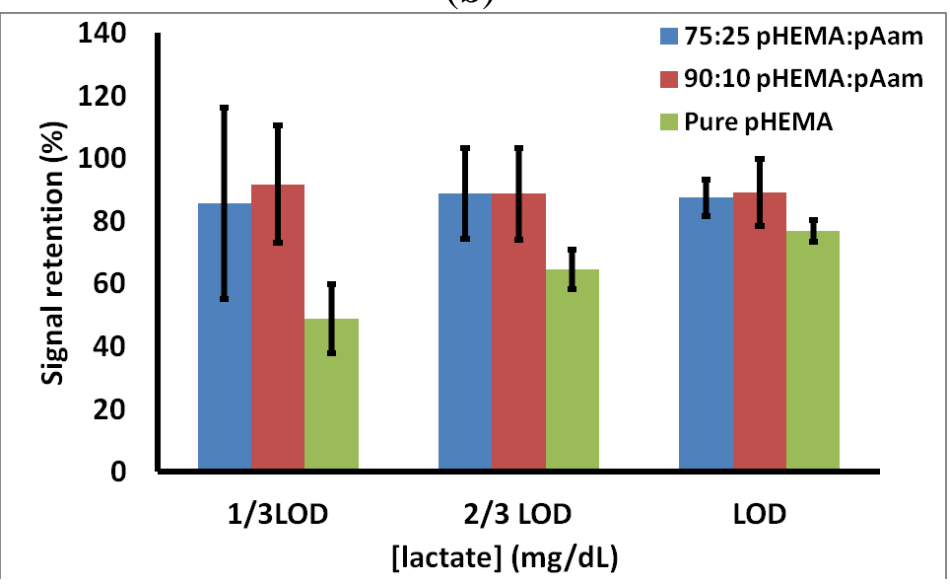

(c)

Figure 4. (a) 90:10 pHEMA:pAam signal retention over 20 cycles (b) \% retention of first cycle signal (c) \% retention of fifth cycle signal. Markers indicate average values, and error bars represent $95 \%$ confidence intervals between measured signal retention for $n=3$ sensors.

Investigation of acute signal loss reveals an initial reduction in emission lifetime seen before a pseudo-stable response is reached, as there is no statistical change in lifetime after the 5th exposure cycle (Figure 4a). This "break-in" could be due to leaching of LOx not covalently bound to the 
hydrogel, though leaching studies done on freshly-prepared sensors showed no evidence of such (data not shown). The likely culprit is chemical and mechanical stresses induced on enzyme during polymerization and swelling during equilibration. These partially unstable proteins quickly denature (within first $2 \mathrm{~h}$ of lactate challenge), leaving remaining LOx to function properly. Figure 4c shows stability of lifetimes of each sensor type at $\mathrm{LOD}_{\text {high }}$ between cycles 5-20. After an initial loss in activity, all co-polymer materials maintained a stable response, with no statistical difference from the 5 th cycle response to the 20th cycle at any of the test lactate concentrations.

These data also suggest a positive relationship between sensor stability and increasing pAam concentration. Both 75:25 pHEMA:pAam and 90:10 pHEMA:pAam experienced statistically similar signal retention throughout the study, while pure pHEMA formulations displayed higher levels of LOx activity loss (about 50\%-70\% retention between cycles 5-20). It follows that gels containing some acrylamide are better suited for retaining LOx activity, most likely due to the hydrophilic nature of pAam that yields an encapsulation more consistent with the enzymes' native environment.

Aside from leaching and mechanical denaturation issues, neither of which were observed in our studies, enzyme activity is the primary issue for sensor stability. It is difficult to quantify enzyme activity within a semisolid medium. Furthermore, it is important to appreciate that, upon hydrogel immobilization, the kinetics of LOx no longer exclusively depend on lactate concentration. The LOx/polymer interface restricts transport, making substrate less available as when in solution. This means that the hydrogel composition will directly determine the rate of lactate delivery to the enzyme; this system requires normalizing conditions for direct comparison. Since each sensor type has a different calculated $\mathrm{LOD}_{\text {high }}$, we chose to expose them to the same normalized concentrations relative to this LOD during the experiment; this resulted in different absolute bulk lactate concentrations but effectively the same lactate flux. Although $\mathrm{LOD}_{\text {high }}$ was used in an attempt to normalize data, we recognize that dissimilar lactate challenges may provide for variable concentrations of substrate near immobilized LOx and therefore different reaction rates.

\section{Conclusions}

In vitro characterization of a novel biosensor designed for lactate detection has been described, using enzyme-oxygen, phosphor sensing chemistry immobilized within three different co-polymer formulations. The findings reveal that modification of co-polymer ratios allows for tunable macro sensor characteristics by controlling substrate diffusion through careful co-polymer selection. This matched expectations based on known hydrophilicity differences between the pHEMA and pAam materials studied, though the magnitude of the gains in oxygen diffusivity were surprisingly low. Furthermore, this tuning in response properties was possible by adjusting lactate diffusion with minimal effect on oxygen diffusion and phosphor sensitivity to oxygen.

While the hydrogel composition affected the transport properties, there was also an apparent change in acute stability that favors the incorporation of at least some acrylamide. Interestingly, sensor stability to repeated lactate challenges does not directly correlate with the diffusion properties studied herein. This suggests further investigation is necessary to understand mechanisms underlying sensor performance loss to allow optimal device design. 
This study also highlights the potential of the enzyme-oxygen phosphor-hydrogel platform, as it is a modification of a previous glucose sensor. By switching the enzyme and adjusting the hydrogel properties, sensitive and stable lactate sensors were successfully developed. The same approach may, in principle, be used to extend the range of analytes to other targets using oxidoreductase enzymes.

\section{Acknowledgments}

The authors gratefully acknowledge Soya Gamsey (PROFUSA) for supplying the palladium (II)-tetramethacrylate-benzoporphyrin used in this study. This work was supported by the National Institutes of Health (Project number 1R01EB016414). The custom optical instrumentation used in this work was jointly developed by PROFUSA, Inc. and McShane, with funding through the NIH Director's Transformative Research Award (TRA) program.

\section{Author Contributions}

Liam P. Andrus prepared all of the samples for analysis, conducted the in vitro diffusion and optical lactate response experiments, and performed the initial data analysis. Rachel Unruh developed the hydrogel formulations used and advised in the current implementation with lactate oxidase. Natalie A. Wisniewski and Michael J. McShane are the project leaders; they conceived of the overall sensor materials and instrumentation design as well as the experimental design for in vitro testing and data analysis. All authors contributed directly to the manuscript draft.

\section{Conflicts of Interest}

Natalie Wisniewski is an employee of PROFUSA, Inc.

\section{References}

1. Gaster, R.S.; Hall, D.A.; Wang, S.X. nanoLAB: An ultraportable, handheld diagnostic laboratory for global health. Lab Chip 2011, 11, 950-956.

2. Ayub, M.; Ivanov, A.; Instuli, E.; Cecchini, M.; Chansin, G.; McGilvery, C.; Hong, J.; Baldwin, G.; McComb, D.; Edel, J.B.; et al. Nanopore/electrode structures for single-molecule biosensing. Electrochim. Acta 2010, 55, 8237-8243.

3. Roy, D.; Cambre, J.N.; Sumerlin, B.S. Future perspectives and recent advances in stimuli-responsive materials. Prog. Polym. Sci. 2010, 35, 278-301.

4. Klonoff, D.C. Continuous Glucose Monitoring: Roadmap for 21st century diabetes therapy. Diabetes Care 2005, 28, 1231-1239.

5. Palleschi, G.; Mascini, M.; Bernardi, L.; Zeppilli, P. Lactate and glucose electrochemical biosensors for the evaluation of the aerobic and anaerobic threshold in runners. Med. Biol. Eng. Comput. 1990, 28, B25-B28.

6. Jansen, T.C.; van Bommel, J.; Bakker, J. Blood lactate monitoring in critically ill patients: A systematic health technology assessment. Crit. Care Med. 2009, 37, 2827-2839. 
7. Zanini, V.P.; de Mishima, B.L.; Solís, V. An amperometric biosensor based on lactate oxidase immobilized in laponite-chitosan hydrogel on a glassy carbon electrode. Application to the analysis of 1-lactate in food samples. Sens. Actuators B Chem. 2011, 155, 75-80.

8. Ekberg, N.R.; Brismar, K.; Malmstedt, J.; Hedblad, M.-A.; Adamson, U.; Ungerstedt, U.; Wisniewski, N. Analyte flux at a biomaterial-tissue interface over time: Implications for sensors for type 1 and 2 diabetes mellitus. J. Diabetes Sci. Technol. 2010, 4, 1063-1072.

9. Rajamand, N.; Ungerstedt, U.; Brismar, K. Subcutaneous microdialysis before and after an oral glucose tolerance test: A method to determine insulin resistance in the subcutaneous adipose tissue in diabetes mellitus. Diabetes Obes. Metab. 2005, 7, 525-535.

10. Nguyen, H.B.; Rivers, E.P.; Knoblich, B.P.; Jacobsen, G.; Muzzin, A.; Ressler, J.A.; Tomlanovich, M.C. Early lactate clearance is associated with improved outcome in severe sepsis and septic shock. Crit. Care Med. 2004, 32, 1637-1642.

11. Matsushita, K.; Williams, E.K.; Mongraw-Chaffin, M.L.; Coresh, J.; Schmidt, M.I.; Brancati, F.L.; Hoogeveen, R.C.; Ballantyne, C.M.; Young, J.H. The association of plasma lactate with incident cardiovascular outcomes: The ARIC study. Am. J. Epidemiol. 2013, 178, 401-409.

12. Nakao, T. Impaired lactate production by skeletal muscle with anaerobic exercise in patients with chronic renal failure. A possible consequence of defective glycolysis in skeletal muscle. Nephron 1982, 31, 111-115.

13. White, N.J. Lactate trumps blood pressure for trauma triage. Sci. Transl. Med. 2015, 7, $278 \mathrm{ec} 43$.

14. Ghosh, A.K. Anaerobic threshold: Its concept and role in endurance sport. Malays. J. Med. Sci. MJMS 2004, 11, 24-36.

15. Heiss, W.-D. The ischemic penumbra: how does tissue injury evolve? Ann. N. Y. Acad. Sci. 2012, 1268, 26-34.

16. Zhao, W.; Ni, Y.; Zhu, Q.; Fu, R.; Huang, X.; Shen, J. Innovative biocompatible nanospheres as biomimetic platform for electrochemical glucose biosensor. Biosens. Bioelectron. 2013, 44, 1-5.

17. Thomas, N.; Lähdesmäki, I.; Parviz, B.A. A contact lens with an integrated lactate sensor. Sens. Actuators B Chem. 2012, 162, 128-134.

18. Chaudhary, A.; McShane, M.J.; Srivastava, R. Glucose response of dissolved-core alginate microspheres: Towards a continuous glucose biosensor. Analyst 2010, 135, 2620-2628.

19. McShane, M.J. Potential for glucose monitoring with nanoengineered fluorescent biosensors. Diabetes Technol. Ther. 2002, 4, 533-538.

20. Cummins, B.M.; Lim, J.; Simanek, E.E.; Pishko, M.V.; Coté, G.L. Encapsulation of a Concanavalin A/dendrimer glucose sensing assay within microporated poly (ethylene glycol) microspheres. Biomed. Opt. Express 2011, 2, 1243-1257.

21. Rassaei, L.; Olthuis, W.; Tsujimura, S.; Sudhölter, E.R.; van den Berg, A. Lactate biosensors: Current status and outlook. Anal. Bioanal. Chem. 2014, 406, 123-137.

22. Clarke, W.L.; Anderson, S.; Farhy, L.; Breton, M.; Gonder-Frederick, L.; Cox, D.; Kovatchev, B. Evaluating the clinical accuracy of two continuous glucose sensors using continuous glucose-error grid analysis. Diabetes Care 2005, 28, 2412-2417.

23. Koschwanez, H.E.; Reichert, W.M. In vitro, in vivo and post explantation testing of glucose-detecting biosensors: Current methods and recommendations. Biomaterials 2007, 28, 3687-3703. 
24. Wentholt, I.M.E.; Hoekstra, J.B.L.; Devries, J. Continuous glucose monitors: The long-awaited watchdogs? Diabetes Technol. Ther. 2007, 9, 399-409.

25. Yu, B.Z.; Ju, Y.M.; West, L.; Moussy, Y.; Moussy, F. An investigation of long-term performance of minimally invasive glucose biosensors. Diabetes Technol. Ther. 2007, 9, 265-275.

26. Malik, B.H.; Coté, G.L. Characterizing dual wavelength polarimetry through the eye for monitoring glucose. Biomed. Opt. Express 2010, 1, 1247-1258.

27. Esenaliev, R.O.; Larin, K.V.; Larina, I.V.; Motamedi, M. Noninvasive monitoring of glucose concentration with optical coherence tomography. Opt. Lett. 2001, 26, 992-994.

28. Chou, I.H.; Benford, M.; Beier, H.T.; Coté, G.L.; Wang, M.; Jing, N.; Kameoka, J.; Good, T.A. Nanofluidic biosensing for $\beta$-amyloid detection using surface enhanced raman spectroscopy. Nano Lett. 2008, 8, 1729-1735.

29. Kazakova, L.; Shabarchina, L.; Anastasova, S.; Pavlov, A.; Vadgama, P.; Skirtach, A.; Sukhorukov, G. Chemosensors and biosensors based on polyelectrolyte microcapsules containing fluorescent dyes and enzymes. Anal. Bioanal. Chem. 2013, 405, 1559-1568.

30. Hu, A.-L.; Liu, Y.-H.; Deng, H.-H.; Hong, G.-L.; Liu, A.-L.; Lin, X.-H.; Xia, X.-H.; Chen, W. Fluorescent hydrogen peroxide sensor based on cupric oxide nanoparticles and its application for glucose and 1-lactate detection. Biosens. Bioelectron. 2014, 61, 374-378.

31. Caspers, P.; Lucassen, G.; Bruining, H.; Puppels, G. Detection of skin lactate by in vivo Raman spectroscopy. In Spectroscopy of Biological Molecules: New Directions; Greve, J., Puppels, G.J., Otto, C., Eds.; Springer: Dordrecht, The Netherlands, 1999; pp. 485-486.

32. McShane, M. Encapsulated probes. In Sensors Based on Nanostructured Materials; Arregui, F.J., Ed.; Springer: New York, NY, USA, 2009; pp. 253-273.

33. Kürner, J.M.; Wolfbeis, O.S.; Klimant, I. Homogeneous luminescence decay time-based assay using energy transfer from nanospheres. Anal. Chem. 2002, 74, 2151-2156.

34. O’Sullivan, P.J.; Burke, M.; Soini, A.E.; Papkovsky, D.B. Synthesis and evaluation of phosphorescent oligonucleotide probes for hybridisation assays. Nucleic Acids Res. 2002, 30, e114.

35. O'Riordan, T.C.; Soini, A.E.; Papkovsky, D.B. Monofunctional derivatives of coproporphyrins for phosphorescent labeling of proteins and binding assays. Anal. Biochem. 2001, 290, 366-375.

36. Smith, A.M.; Mancini, M.C.; Nie, S. Bioimaging: Second window for in vivo imaging. Nat Nano 2009, 4, 710-711.

37. Lockridge, O.; Massey, V.; Sullivan, P.A. Mechanism of action of the flavoenzyme lactate oxidase. J. Biol. Chem. 1972, 247, 8097-8106.

38. Hoffman, A.S. Hydrogels for biomedical applications. Adv. Drug Deliv. Rev. 2012, 64, $18-23$.

39. Arciola, C.R.; Maltarello, M.C.; Cenni, E.; Pizzoferrato, A. Disposable contact lenses and bacterial adhesion. In vitro comparison between ionic/high-water-content and non-ionic/low-water-content lenses. Biomaterials 1995, 16, 685-690.

40. Ševčík, S.; Vacík, J.; Chmelíková, D.; Smetana, K., Jr. Surface alkaline hydrolysis of 2-hydroxyethyl methacrylate gels. J. Mater. Sci. Mater. Med. 1995, 6, 505-509.

41. Mohapatra, R.; Ray, D.; Swain, A.K.; Pal, T.K.; Sahoo, P.K. Release study of alfuzosin hydrochloride loaded to novel hydrogel P(HEMA-co-AA). J. Appl. Polym. Sci. 2008, 108, 380-386. 
42. Jaiswal, M.; Koul, V. Assessment of multicomponent hydrogel scaffolds of poly(acrylic acid-2hydroxy ethyl methacrylate)/gelatin for tissue engineering applications. J. Biomater. Appl. 2013, 27, 848-861.

43. Ramalingam, N.; Natarajan, T.S.; Rajiv, S. Development and characterization of electrospun poly(2-hydroxy ethyl methacrylate) for tissue engineering applications. Adv. Polym. Technol. 2013, 32 .

44. Montheard, J.-P.; Chatzopoulos, M.; Chappard, D. 2-Hydroxyethyl methacrylate (HEMA): Chemical properties and applications in biomedical fields. J. Macromol. Sci. Part C 1992, 32, 1-34.

45. Kochkodan, V.M.; Hilal, N.; Goncharuk, V.V.; Al-Khatib, L.; Levadna, T.I. Effect of the surface modification of polymer membranes on their microbiological fouling. Colloid J. 2006, 68, 267-273.

46. Goli, K.K.; Rojas, O.J.; Genzer, J. Formation and antifouling properties of amphiphilic coatings on polypropylene fibers. Biomacromolecules 2012, 13, 3769-3779.

47. Peppas, N.A.; Moynihan, H.J.; Lucht, L.M. The structure of highly crosslinked poly(2-hydroxyethyl methacrylate) hydrogels. J. Biomed. Mater. Res. 1985, 19, 397-411.

48. Raymond, S. Acrylamide gel electrophoresis. Ann. N. Y. Acad. Sci. 1964, 121, 350-365.

49. Akroyd, P. Acrylamide-gel electrophoresis of [beta]-lactoglobulins stored in solutions at $\mathrm{pH}$ 8.7. Nature 1965, 208, 488-489.

50. Ramaraj, B.; Radhakrishnan, G. Hydrogel capsules for sustained drug release. J. Appl. Polym. Sci. 1994, 51, 979-988.

51. Roberts, J.; Collier, B.B.; McShane, M.J. Incorporation of optical enzymatic sensing chemistry into biocompatible hydrogels. In Proceedings of the 2011 IEEE on Sensors, Limerick, Irelan, 28-31 October 2011; pp. 1245-1248.

52. Roberts, J.R.; Park, J.; Helton, K.; Wisniewski, N.; McShane, M.J. Biofouling of polymer hydrogel materials and its effect on diffusion and enzyme-based luminescent glucose sensor functional characteristics. J. Diabetes Sci. Technol. 2012, 6, 1267-1275.

53. Markvicheva, E.A.; Tkachuk, N.E.; Kuptsova, S.V.; Dugina, T.N.; Strukova, S.M.; Kirssh, Y.E.; Zubov, V.P.; Rumish, L.D. Stabilization of proteases by entrapment in a new composite hydrogel. Appl. Biochem. Biotechnol. 1996, 61, 75-84.

54. Zhu, X.; Ma, Y.; Zhao, C.; Lin, Z.; Zhang, L.; Chen, R.; Yang, W. A mild strategy to encapsulate enzyme into hydrogel layer grafted on polymeric substrate. Langmuir 2014, 30, 15229-15237.

55. Baker, D.A.; Corkhill, P.H.; Ng, C.O.; Skelly, P.J.; Tighe, B.J. Synthetic hydrogels: 2. Copolymers of carboxyl-, lactam- and amide-containing monomers-Structure/property relationships. Polymer 1988, 29, 691-700.

56. Garg, S.K.; Voelmle, M.; Gottlieb, P.A. Time lag characterization of two continuous glucose monitoring systems. Diabetes Res. Clin. Pract. 2010, 87, 348-353.

57. Kamath, A.; Mahalingam, A.; Brauker, J. Analysis of time lags and other sources of error of the DexCom SEVEN continuous glucose monitor. Diabetes Technol. Ther. 2009, 11, 689-695.

58. Garjonyte, R.; Yigzaw, Y.; Meskys, R.; Malinauskas, A.; Gorton, L. Prussian Blue- and lactate oxidase-based amperometric biosensor for lactic acid. Sens. Actuators B Chem. 2001, 79, 33-38. 
59. Palmisano, F.; Centonze, D.; Zambonin, P.G. An in situ electrosynthesized amperometric biosensor based on lactate oxidase immobilized in a poly-o-phenylenediamine film: Determination of lactate in serum by flow injection analysis. Biosens. Bioelectron. 1994, 9, 471-479.

60. Ibupoto, Z.H.; Shah, S.M.; Khun, K.; Willander, M. Electrochemical L-lactic acid sensor based on immobilized $\mathrm{ZnO}$ nanorods with lactate oxidase. Sensors 2012, 12, 2456-2466.

61. Marquette, C.A.; Leca, B.D.; Blum, L.J. Electrogenerated chemiluminescence of luminol for oxidase-based fibre-optic biosensors. Luminescence 2001, 16, 159-165.

(C) 2015 by the authors; licensee MDPI, Basel, Switzerland. This article is an open access article distributed under the terms and conditions of the Creative Commons Attribution license (http://creativecommons.org/licenses/by/4.0/). 